\title{
Spondyloarthritis: Does no clinical enthesitis mean no enthesitis at all? The role of ultrasonography in everyday practice
}

\author{
Daniela-Raluca Decianu', Violeta-Claudia Bojinca ${ }^{1,2}$, Mihai Bojinca ${ }^{2,3}$, \\ Iuliana-Raluca Gheorghe ${ }^{4}$, Andra-Rodica Balanescu ${ }^{1,2}$, Ruxandra Ionescu ${ }^{1,2}$ \\ 1"Sfanta Maria" Clinical Hospital, Bucharest, Romania \\ 2"Carol Davila" University of Medicine and Pharmacy, Bucharest, Romania \\ 3"Dr. I. Cantacuzino" Clinical Hospital, Bucharest, Romania \\ ${ }^{4}$ Department of Marketing and Medical Technology, "Carol Davila" University of Medicine and Pharmacy, \\ Bucharest, Romania
}

\begin{abstract}
Objectives. The purpose of this paper is to assess the frequency of ultrasonography detected enthesitis in spondyloarthritis (SpA) patients as well as it's correlation to reported pain and disease activity.

Methods. $80 \mathrm{SpA}$ patients were evaluated clinically (medical history, clinical examination, inflammatory markers, clinical and composite indices) and using musculoskeletal ultrasonography (MSUS). The conducted evaluation focused on 16 entheseal sites for each patient, reaching a total of 1280 entheses. The medical history form included questions regarding present or past spontaneous pain and the clinical examination, which evaluated entheseal pain upon pressure (digital pressure on the enthesis overlying skin). Two Esaote My Lab machines with 6-12/8-18 MHz linear probes were used. One clinician/ultrasonographer performed all evaluations, in order to avoid interobserver variability.

Results. Using musculoskeletal ultrasonography, up to $54.68 \%$ of the asymptomatic entheses in patients with axial/ peripheral SpA were found with either GS and/or PD abnormalities.

The tendons with the highest rates of ultrasound detected entesitis were the Achilles, followed by the quadriceps, both in gray scale (GS) $(76.25 \%, 56.25 \%$ respectively) and power Doppler (PD) $(24.4 \%, 20 \%$ respectively), while the least affected were the proximal patellar (20.65\% GS, 8.15\% PD) and flexor tendons (19.4\% GS, 9.4\% PD). Conclusions. Although it has a leading role in the pathogenesis of SpA, enthesitis is partially overlooked in everyday practice. This paper highlights the high percentages of both GS and PD entheseal abnormalities, both in peripheral and axial SpA.

The low correlation between reported peripheral pain, especially in axial SpA, and MSUS signs of enthesitis proves the need for an imagistic enthesitis score in the diagnostic process, in order to establish the full extent of the disease and to personalize the treatment, considering the high and rising range of therapies to choose from.
\end{abstract}

Keywords: spondyloarthritits, axial, peripheral, enthesitis, musculoskeletal ultrasonography

\section{INTRODUCTION}

The term spondyloarthritis ( $\mathrm{SpA}$ ) covers a heterogeneous family of inflammatory diseases with several common features, such as the axial, sacroiliac and peripheral joint involvement, including arthritis, enthesitis and dactilitis, but also trigger and genetic factors, like mechanical stress, infectious diseases or gut dysbiosis. Between the range of different SpA phenotypes, of more interest in Rheumatology are those with primarily skeletal involvement, such as axial (ax) SpA (radiographic axial SpA (r-ax-SpA) ankylosing spondylitis (AS) and nonradiographic axial SpA (nr-ax-SpA)) and peripheral SpA.

No matter the phenotype or the trigger factor, entheseal inflammation is the common ground in the pathogenesis of SpA. Enthesitis further leads to osteitis, periostitis and osteoproliferation, present in varied proportions in all SpA [1,2]. Despite being a cen- 
tral feature in SpA and the number of scores elaborated in order to standardize it is high, enthesitis remains underdiagnosed in everyday practice.

Clinically, the chronic lower back pain is the most common symptom of SpA. Other features, such as oligoarthritis and dactilitis are usually accompanied by morning stiffness and fatigue. Usually, SpA presents periods of flare and remission or low disease activity and, progressively, evolves towards a reduced mobility. The definition of flare in SpA is still a work in progress, being based on pain, Bath AS Disease Activity Index (BASDAI), $\mathrm{C}$ reactive protein (CRP) and varied combinations, such as the composite index AS Disease Activity Score (ASDAS) [3-8].

AxSpA has an estimated incidence of $0.5 \%$, with a prevalence of 30 per 10.000 persons, or up to 1 per 200 persons and even $1.5 \%$ (all SpA) in different studies. The progression from nr-ax-SpA to r-ax-SpA is estimated to $5.1 \%$ in 5 years and $19 \%$ in 10 years. Even with radiographic changes that occur only after several years, and despite not only having a large number of diagnosed patients, but also a wide range of research, published studies and elaboration of good practices, the general goal of early diagnosis of SpA remains mostly under discussed. Consequently, this leads to the "lost tribe" of patients with structural changes and functional deficit when diagnosed [1,911]. The presence of baseline structural damage, as well as the male sex, active disease state, higher inflammatory markers (raised CRP and/or ESR) and smoking, include these patients in a high-risk group for progression [12].

In $\mathrm{SpA}$, the enthesis has benefited from a high level of interest in the last two decades, with rising amount of knowledge on the subject and even the terminology evolving from enthesis to entheseal organ and synovio-entheseal complex [13].

The entheses are fibrocartilaginous structures, arising at the insertion sites of tendons, ligaments, joint capsules or nails. The insertional fibrocartilages is generally prone to microdamage, but avascular and lacking immune cells. SpA patients develop a disproportional degree of inflammation after physiological mechanical stress, with a vessel tissue repair responses and vessel ingrowth activation of pro-inflammatory cytokines tumor necrosis factor (TNF) $\alpha$ and interleukin (IL) 17, accompanied by an inflow of innate immune cells $[3,13]$.

TNF, IL-17 and IL-23-mediated immune pathways have a leading role in triggering enthesitis, while the proliferation process is mediated by IL-22.
Gut and exogenous bacteria could also trigger and unleash an immune reaction, with autoimmunity aiming for fibrocartilage peptides, such as the versican or the bone morphogenetic protein (BMP), in particular in patients with a genetic predisposition, given by the presence of the HLA-B27 antigen. Also, triggered by TNF- $\alpha$ and IL-1, BMP-7 mostly, accompanied by BMP-2 and BMP- 6 are involved in the new bone formation that follows the entheseal inflammation, leading to peripheral and axial bony spurs, enthesophytes and syndesmophytes. Therefore, enthesitis seems to be a diffuse process in SpA, as has been proved by advanced imaging techniques and biopsy results [14-18].

The diagnostic delay in SpA and, especially, in AS, is reported as 8-10years and under 6 years in more recent publications $[19,20]$. The determining factor in the more early diagnosis is most probably the use of imaging, in this case, the Magnetic Resonance Imaging (MRI) in the diagnosis of ax-SpA and the use of Assessment of SpondyloArthritis International Society (ASAS) classification criteria [21,22].

Following this example, imaging of the entheses could be the key for a more early diagnosis of not only periphereal, but also ax-SpA. For now, enthesitis remains relatively overlooked and underdiagnosed in the clinical everyday practice, despite being a central feature in SpA and despite the number of scores elaborated in order to standardize it.

Of course, MRI is the gold standard, but still implies high costs, long scanning duration and magnetic limitations, ultrasound being a convenient and comfortable bedside tool for detecting and monitoring enthesitis, with on spot results and fewer limitations, related mostly to the ability of viewing only relatively superficial structures. Musculoskeletal ultrasonography (MSUS) provides high fidelity images of the entheseal structure and detailed information regarding the vascularity, equivalent to the degree of local inflammation and therefore, disease activity; these findings having higher sensibility and specificity for SpA than a physical evaluation alone [23-25].

The primary objective of this study is to evaluate the frequency of clinical versus ultrasound detected enthesitis in Romanian patients with highly active $\mathrm{SpA}$. A secondary objective is to determine the correlation between the presence of MSUS grey scale (GS) and/or power Doppler (PD) entheseal abnormalities and the disease activity, biologic inflammatory markers and to clinical pain, either declared dur- 
ing a detailed, targeted medical history or detected in a thorough clinical exam.

\section{METHODS}

\section{Design and patients}

The study included 80 patients fulfilling the ASAS criteria for either axial or peripheral SpA [16,17], in a random order of presentation to the Out-patient Clinic or the Department of Internal Medicine and Rheumatology of the "Sf. Maria" Clinical Hospital or "Dr. I. Cantacuzino" Clinical Hospital, in Bucharest. Patients were recruited between December 2018 and August 2020. All patients suffered from highly active disease (BASDAI $>4$, ASDAS $>2.1$ ) and they were all naive to biological disease-modifying antirheumatic drugs (bDMARD). The 16 entheseal sites targeted in this study were the insertions of: Achilles tendon, plantar fascia, qvadriceps tendon, proximal and distal patellar tendon, triceps tendon, extensor and flexor tendons of the hand, all evaluated on both sides, right and left. The exclusion criteria consisted of age below 18 years, overlap or other chronic inflammatory rheumatic disease, bDMARD therapy, history of knee/ankle/elbow surgery.

Medications that could influence the ultrasound findings were allowed, as for instance oral glucocorticoids, if less than $10 \mathrm{mg}$ prednisone equivalent per day and stable in the prior month; non-steroidal anti-inflammatory drugs (NSAIDs), if stable in the prior week, conventional synthetic DMARDs (csDMARDs: sulfasalazine, methotrexate, leflunomide), if stable in the prior month.

The study was approved by the Local Ethics Committee and all patients signed a written informed consent that they agree to take part in this research.

\section{Patient-reported outcomes}

The patient's global assessment of disease activity was evaluated on a $10 \mathrm{~cm}$ visual analogue scale (VAS).

The reported pain upon pressure was induced by applying digital pressure (policies, until nail whitening) on every entheseal site's overlying skin. Careful examination of the adjacent joint excluded referred pain.

\section{Clinical examination}

Spontaneous pain was evaluated by addressing the question „Do you or did you ever feel pain in this particular spot?" (finger-pointed by the examiner, without touching the overlying skin) for each of the 16 entheseal sites. Pain described after a local traumatic event was disregarded, therefore not noted as a symptom of enthesitis. The responses were noted as 0 „absence of pain, ever" or 1 ,presence of pain, in the present or in the past".

Thus, medical history and clinical examination forms were completed, including fatigue, axial and peripheral joint pain and stiffness and was also added the digital pressure-induced entheseal pain. The responses were noted as 0 ,absence of pain during enthesis pressure" or 1 ,presence of pain during enthesis pressure".

All clinical examinations were performed by the same rheumatologist, simultaneously with the ultrasound examination.

\section{Ultrasound}

Esaote My Lab machines with 6-12 $\mathrm{MHz}$ and 8-18 MHz linear probes were used.

The same rheumatologist, EULAR Level I certified (June 2018, Amsterdam), performed and interpreted all MSUS examinations. All abnormalities have been confirmed in a second perpendicular plane.

Grey scale (GS) abnormalities consisted of: insertional bone erosion, modified tendon structure, increased tendon width and insertional tendon calcification. The presence of any of the above was noted as a GS-modified enthesis. Considering the superficial location of the studied entheses, the ultrasound evaluation was performed using a high frequency (12-15 $\mathrm{MHz}$ ).

Power Doppler (PD) was evaluated with a low wall filter (1), a pulse repetition frequency (PRF) of 500 to $1,000 \mathrm{~Hz}$, while lowering the gain from a high, artefacted value, until the disappearance of the last spot below the cortical line. The identification of any grade of pathological PD signal was noted as present PD signal. A thick layer of gel was always applied on the probe, in order to avoid small vessel compression, leading to a false negative PD signal.

\section{Disease activity and acute phase reactants}

The disease activity was measured using a clinical score, BASDAI and a composite index, ASDAS, in order to ensure higher reliability.

Laboratory tests included C-reactive protein (CRP; normal $<5 \mathrm{mg} / 1$; latex-immunoturbidimetry method using commercially-available kits) and ESR (normal $<20 \mathrm{~mm} / \mathrm{h}$; Westergren method). 

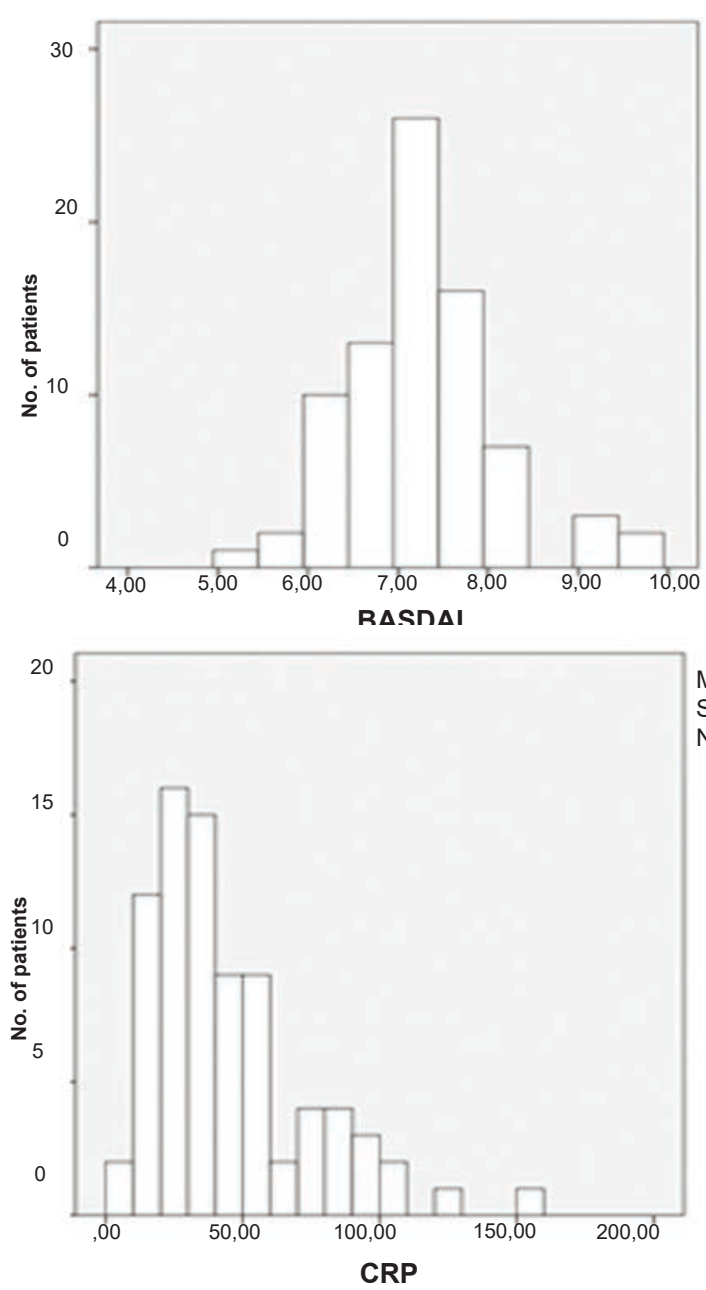

FIGURE 1ABCD. Number of patients by characteristics

\section{Statistics}

For the distribution of the quantitative variables (i.e., age), the mean and the standard deviation were used, whereas for the distribution of the qualitative variables, the frequency was employed. Moreover, the performance of the ultrasound examination (GS, PD) in comparison to the clinical examination (spontaneous pain, elicited pain) was ascertained with the Cohen's $k$ value, for which the strength of agreement has the following interpretation $<0.2=$ poor measurement agreement, $0.21-0.40=$ fair measurement agreement, 0.41-0.60 = moderate measurement agreement, 0.61$0.80=$ good measurement agreement,$>0.80=$ excellent measurement agreement [26].

All tests were considered significant at a $p$ threshold lower than 0.05 . Data was collected and analyzed in IBM SPSS version 20.

\section{RESULTS}

\section{General and SpA characteristics}

General and SpA characteristics are presented in table 1-6 and Figure 1ABCD.
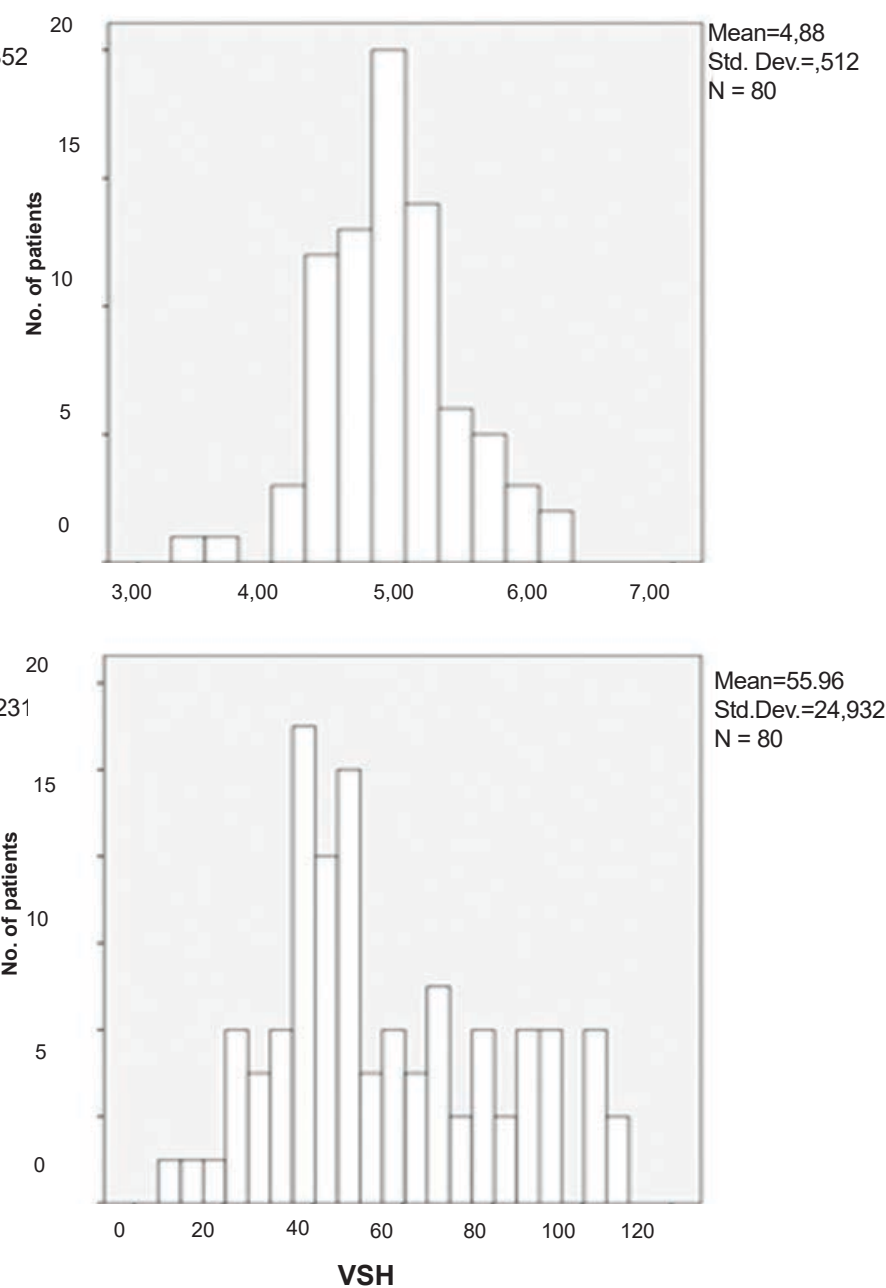

TABLE 1. General and SpA characteristics $(n=80)$

\begin{tabular}{|c|c|}
\hline $\operatorname{men}(\mathrm{n} ; \%)$ & $51(63.8 \%)$ \\
\hline $\operatorname{age}(\mathrm{y} ;$ mean $(\mathrm{SD}))$ & $43.13(12.35)$ \\
\hline smoking $(\mathrm{n} ; \%)$ & $16(20 \%)$ \\
\hline urban dwelling $(\mathrm{n} ; \%)$ & $60(75 \%)$ \\
\hline $\operatorname{axial} \operatorname{SpA}(\mathrm{n} ; \%)$ & $32(40 \%)$ \\
\hline peripheral SpA (n; \%) & $30(37.5 \%)$ \\
\hline axial+peripheral SpA (n; \%) & $18(22.5 \%)$ \\
\hline SpA duration (mos; median (SD)) & $67.28(85.69)$ \\
\hline dactylitis ( $\mathrm{n} ; \%)$ & $9(11.3 \%)$ \\
\hline uveitis ( $\mathrm{n} ; \%)$ & $7(8.8 \%)$ \\
\hline BASDAI (mean (SD)) & $7.24(0.85)$ \\
\hline ASDAS (mean (SD)) & $4.88(0.51)$ \\
\hline$C R P(\mathrm{mg} / \mathrm{l} ;$ mean (SD) & $44.97(29.23)$ \\
\hline$E S R(\mathrm{~mm} / \mathrm{h} ;$ mean $(\mathrm{SD}))$ & $55.96(24.93)$ \\
\hline HLA-B27 positive ( $\mathrm{n} ; \%)$ & $69(86.3 \%)$ \\
\hline $\operatorname{csDMARDs}(\mathrm{n} ; \%)$ & $44(55 \%)$ \\
\hline glucocorticoids $(\mathrm{n} ; \%)$ & $22(27.5 \%)$ \\
\hline
\end{tabular}

ASDAS - Ankylosing Spondylitis Disease Activity Score; BASDAI - Bath Ankylosing Spondylitis Disease Activity Index; CRP - C-reactive protein (normal < $5 \mathrm{mg} / \mathrm{l}$ ); csDMARDs - conventional synthetic disease-modifying anti-rheumatic drugs; ESR - erythrocyte sedimentation rate (normal $<20 \mathrm{~mm} / \mathrm{h}$ ); mos - months; SD - standard deviation; SpA - spondyloarthritis; $y$ - years 
TABLE 2. Clinical and ultrasound enthesitis prevalence by site $(n=80)$

\begin{tabular}{|c|c|c|c|c|}
\hline \multirow[t]{2}{*}{ 1. Clinical involvement } & \multicolumn{2}{|c|}{ symptomatic (spontaneous) } & \multicolumn{2}{|c|}{ symptomatic (examination) } \\
\hline & right & left & right & left \\
\hline Achilles' tendon & $24(30 \%)$ & $22(27.5 \%)$ & $47(58.8 \%)$ & $49(61.3 \%)$ \\
\hline plantar fascia & $9(11.3 \%)$ & $10(12.5 \%)$ & $14(17.5 \%)$ & $13(16.3 \%)$ \\
\hline quadriceps tendon & $25(31.3 \%)$ & $27(33.8 \%)$ & $38(47.5 \%)$ & $36(45 \%)$ \\
\hline proximal patellar ten-don & $4(5 \%)$ & $2(2.5 \%)$ & $10(12.5 \%)$ & $8(10 \%)$ \\
\hline distal patellar tendon & $14(17.5 \%)$ & $14(17.5 \%)$ & $25(31.3 \%)$ & $24(30 \%)$ \\
\hline triceps tendon & $5(6.3 \%)$ & $3(3.8 \%)$ & $17(21.3 \%)$ & $11(13.8 \%)$ \\
\hline flexor tendons & $5(6.3 \%)$ & $4(5 \%)$ & $8(10 \%)$ & $9(11.3 \%)$ \\
\hline extensor tendons & $6(7.5 \%)$ & $8(10 \%)$ & $13(16.3 \%)$ & $12(15 \%)$ \\
\hline \multirow[t]{2}{*}{ 2. Ultrasound involvement } & \multicolumn{2}{|c|}{ GS involvement } & \multicolumn{2}{|c|}{ PD involvement } \\
\hline & right & left & right & left \\
\hline Achilles' tendon & $62(77.5 \%)$ & $60(75 \%)$ & $18(22.5 \%)$ & $21(26.3 \%)$ \\
\hline plantar fascia & $20(25 \%)$ & $18(22.5 \%)$ & $8(10 \%)$ & $6(7.5 \%)$ \\
\hline quadriceps tendon & $46(57.5 \%)$ & $44(55 \%)$ & $16(20 \%)$ & $16(20 \%)$ \\
\hline proximal patellar ten-don & $18(22.5 \%)$ & $15(18.8 \%)$ & $8(10 \%)$ & $5(6.3 \%)$ \\
\hline distal patellar tendon & $39(48.8 \%)$ & $37(46.3 \%)$ & $14(17.5 \%)$ & $13(16.3 \%)$ \\
\hline triceps tendon & $27(33.8 \%)$ & $21(26.3 \%)$ & $7(8.8 \%)$ & $6(7.5 \%)$ \\
\hline flexor tendons & $16(20 \%)$ & $15(18.8 \%)$ & $8(10 \%)$ & $7(8.8 \%)$ \\
\hline extensor tendons & $22(27.5 \%)$ & $22(27.5 \%)$ & $15(18.8 \%)$ & $15(18.8 \%)$ \\
\hline
\end{tabular}

GS-grey scale; PD - power Doppler

\section{Enthesitis prevalence}

TABLE 3. Spontaneously symptomatic enthesitis prevalence by SpA type and site $(n=80)$

\begin{tabular}{|c|c|c|c|c|c|c|}
\hline & \multicolumn{6}{|c|}{ Symptomatic (spontaneous) } \\
\hline & \multicolumn{2}{|c|}{ axial $S p A$} & \multicolumn{2}{|c|}{ peripheral SpA } & \multicolumn{2}{|c|}{ axial+peripheral SpA } \\
\hline & right & left & right & left & right & left \\
\hline Achilles' tendon & $10(31.3 \%)$ & 7 (21.9\%) & $8(26.7 \%)$ & $11(36.7 \%)$ & $6(33.3 \%)$ & $4(22.2 \%)$ \\
\hline plantar fascia & $3(9.4 \%)$ & $3(9.4 \%)$ & $3(10 \%)$ & $3(10 \%)$ & $3(16.7 \%)$ & $4(22.2 \%)$ \\
\hline quadriceps tendon & $7(21.9 \%)$ & $8(25 \%)$ & $11(36.7 \%)$ & $11(36.7 \%)$ & $7(38.9 \%)$ & $8(44.4 \%)$ \\
\hline $\begin{array}{r}\text { proximal patellar } \\
\text { ten-don }\end{array}$ & $3(9.4 \%)$ & $2(6.3 \%)$ & $1(3.3 \%)$ & 0 & 0 & 0 \\
\hline $\begin{array}{r}\text { distal patellar } \\
\text { tendon }\end{array}$ & $4(12.5 \%)$ & $3(9.4 \%)$ & $9(30 \%)$ & $9(30 \%)$ & $1(5.6 \%)$ & $2(11.1 \%)$ \\
\hline triceps tendon & 0 & 0 & $3(10 \%)$ & $2(6.7 \%)$ & $2(11.1 \%)$ & $1(5.6 \%)$ \\
\hline flexor tendons & $1(3.1 \%)$ & $1(3.1 \%)$ & $1(3.3 \%)$ & $2(6.7 \%)$ & $3(16.7 \%)$ & $1(5.6 \%)$ \\
\hline extensor tendons & $3(9.4 \%)$ & $3(9.4 \%)$ & $1(3.3 \%)$ & $2(10 \%)$ & $2(11.1 \%)$ & $2(11.1 \%)$ \\
\hline
\end{tabular}

GS - grey scale; $P D$ - power Doppler

TABLE 4. Symptomatic by examination enthesitis prevalence by SpA type and site $(n=80)$

\begin{tabular}{|c|c|c|c|c|c|c|}
\hline & \multicolumn{6}{|c|}{ Symptomatic (examination) } \\
\hline & \multicolumn{2}{|c|}{ axial $S p A$} & \multicolumn{2}{|c|}{ peripheral SpA } & \multicolumn{2}{|c|}{ axial+peripheral SpA } \\
\hline & right & left & right & left & right & left \\
\hline Achilles' tendon & $18(56.3 \%)$ & $21(65.6 \%)$ & $16(53.3 \%)$ & $16(53.3 \%)$ & $13(72.2 \%)$ & $12(66.7 \%)$ \\
\hline plantar fascia & $3(9.4 \%)$ & $4(12.5 \%)$ & $8(26.7 \%)$ & $5(16.7 \%)$ & $3(16.7 \%)$ & $4(22.2 \%)$ \\
\hline quadriceps tendon & $12(37.5 \%)$ & $10(31.3 \%)$ & $18(60 \%)$ & $15(50 \%)$ & $8(44.4 \%)$ & $11(61.1 \%)$ \\
\hline $\begin{array}{r}\text { proximal patellar } \\
\text { tendon }\end{array}$ & $7(21.9 \%)$ & $5(15.6 \%)$ & $2(6.7 \%)$ & $3(10 \%)$ & $1(5.6 \%)$ & 0 \\
\hline $\begin{array}{r}\text { distal patellar } \\
\text { tendon }\end{array}$ & $8(25 \%)$ & $4(12.5 \%)$ & $14(46.7 \%)$ & $16(53.3 \%)$ & $3(16.7 \%)$ & $4(22.2 \%)$ \\
\hline triceps tendon & $5(15.6 \%)$ & $3(9.4 \%)$ & $6(20 \%)$ & $4(13.3 \%)$ & $6(33.3 \%)$ & $4(22.2 \%)$ \\
\hline flexor tendons & $1(3.1 \%)$ & $3(9.4 \%)$ & $2(6.7 \%)$ & $5(16.7 \%)$ & $5(27.8 \%)$ & $1(5.6 \%)$ \\
\hline extensor tendons & $4(12.5 \%)$ & $3(9.4 \%)$ & $5(16.7 \%)$ & $6(20 \%)$ & $4(22.2 \%)$ & $3(16.7 \%)$ \\
\hline
\end{tabular}


TABLE 5. GS ultrasound enthesitis prevalence by SpA type and site $(n=80)$

\begin{tabular}{|c|c|c|c|c|c|c|}
\hline & \multicolumn{6}{|c|}{ GS involvement } \\
\hline & \multicolumn{2}{|c|}{ axial SpA } & \multicolumn{2}{|c|}{ peripheral SpA } & \multicolumn{2}{|c|}{ axial+peripheral SpA } \\
\hline & right & left & right & left & right & left \\
\hline Achilles' tendon & $21(65.6 \%)$ & $23(71.9 \%)$ & $24(80 \%)$ & $22(73.3 \%)$ & $17(94.4 \%)$ & $15(83.3 \%)$ \\
\hline plantar fascia & 7 (21.9\%) & $4(12.5 \%)$ & $10(33.3 \%)$ & $9(30 \%)$ & $3(16.7 \%)$ & $5(27.8 \%)$ \\
\hline quadriceps tendon & $14(43.8 \%)$ & $14(43.8 \%)$ & $21(70 \%)$ & $17(56.7 \%)$ & $11(61.1 \%)$ & $13(72.2 \%)$ \\
\hline $\begin{array}{r}\text { proximal patellar } \\
\text { tendon }\end{array}$ & $9(28.1 \%)$ & 7 (21.9\%) & $6(20 \%)$ & $6(20 \%)$ & $3(16.7 \%)$ & $2(11.1 \%)$ \\
\hline $\begin{array}{r}\text { distal patellar } \\
\text { tendon }\end{array}$ & $12(37.5 \%)$ & $9(28.1 \%)$ & $18(60 \%)$ & $20(66.7 \%)$ & $9(50 \%)$ & $8(44.4 \%)$ \\
\hline triceps tendon & $8(25 \%)$ & $6(18.8 \%)$ & $10(33.3)$ & $9(30 \%)$ & $9(50 \%)$ & $6(33.3 \%)$ \\
\hline flexor tendons & $3(9.4 \%)$ & $6(18.8 \%)$ & $7(23.3 \%)$ & $8(26.7 \%)$ & $6(33.3 \%)$ & $1(5.6 \%)$ \\
\hline extensor tendons & $8(25 \%)$ & $9(28.1 \%)$ & $9(30 \%)$ & $10(33.3 \%)$ & $5(27.8 \%)$ & $3(16.7 \%)$ \\
\hline
\end{tabular}

GS-grey scale

TABLE 6. PD ultrasound enthesitis prevalence by SpA type and site $(n=80)$

\begin{tabular}{|c|c|c|c|c|c|c|}
\hline & \multicolumn{6}{|c|}{ PD involvement } \\
\hline & \multicolumn{2}{|c|}{ axial SpA } & \multicolumn{2}{|c|}{ peripheral SpA } & \multicolumn{2}{|c|}{ axial+peripheral SpA } \\
\hline & right & left & right & left & right & left \\
\hline Achilles' tendon & $6(18.8 \%)$ & $5(15.6 \%)$ & $10(33.3 \%)$ & $12(40 \%)$ & $2(11.1 \%)$ & $4(22.2 \%)$ \\
\hline plantar fascia & $1(3.1 \%)$ & $1(3.1 \%)$ & $5(16.7 \%)$ & $4(13.3 \%)$ & $2(11.1 \%)$ & $1(5.6 \%)$ \\
\hline quadriceps tendon & $3(9.4 \%)$ & $4(12.5 \%)$ & $8(26.7 \%)$ & $8(26.7 \%)$ & $5(27.8 \%)$ & $4(22.2 \%)$ \\
\hline $\begin{array}{r}\text { proximal patellar } \\
\text { tendon }\end{array}$ & $4(12.5 \%)$ & $3(9.4 \%)$ & $4(13.3 \%)$ & $2(6.7 \%)$ & 0 & 0 \\
\hline $\begin{array}{r}\text { distal patellar } \\
\text { tendon }\end{array}$ & $2(6.3 \%)$ & $4(12.5 \%)$ & $9(30 \%)$ & $8(26.7 \%)$ & $3(16.7 \%)$ & $1(5.6 \%)$ \\
\hline triceps tendon & 0 & $4(12.5 \%)$ & $4(13.3 \%)$ & $1(3.3 \%)$ & $3(16.7 \%)$ & $1(5.6 \%)$ \\
\hline flexor tendons & $1(3.1 \%)$ & $2(6.3 \%)$ & $2(6.7 \%)$ & $4(13.3 \%)$ & $5(27.8 \%)$ & $1(5.6 \%)$ \\
\hline extensor tendons & $4(12.5 \%)$ & $5(15.6 \%)$ & $6(20 \%)$ & $9(30 \%)$ & $5(27.8 \%)$ & $1(5.6 \%)$ \\
\hline
\end{tabular}

PD-power Doppler

\section{Correlations with clinical SpA variables}

In $\mathrm{SpA}$, the best performance of clinical and ultrasound examination was observed in the evaluation of the flexor tendons of the hand (Table 7), with strong and excellent agreement between the two methods. Conversely, the lowest performance of clinical and ultrasound examination was noticed for Achilles and distal patellar tendons, with low agreement indices

TABLE 7. Performance and agreement of clinical and US evaluation of enthesitis $(n=80)$

\begin{tabular}{|l|c|c|c|c|c|}
\hline Enthesis & Side & Variables & $\begin{array}{l}\text { Measurement } \\
\text { agreement }\end{array}$ & kappa & $\mathbf{p}$ \\
\hline \multirow{4}{*}{$\begin{array}{l}\text { Achilles } \\
\text { tendon }\end{array}$} & \multirow{3}{*}{$\mathrm{R}$} & $\mathrm{GS}-\mathrm{SP}$ & poor & 0.13 & 0.047 \\
\cline { 3 - 6 } & & $\mathrm{PD}-\mathrm{SP}$ & fair & 0.29 & 0.007 \\
\cline { 3 - 6 } & & $\mathrm{GS}-\mathrm{EP}$ & moderate & 0.41 & 0.001 \\
\cline { 3 - 6 } & \multirow{3}{*}{$\mathrm{LD}-\mathrm{EP}$} & fair & 0.247 & 0.003 \\
\cline { 3 - 6 } & $\mathrm{L}$ & $\mathrm{PD}-\mathrm{SP}$ & fair & 0.396 & 0.001 \\
\cline { 3 - 6 } & & $\mathrm{PD}-\mathrm{EP}$ & fair & 0.352 & 0.001 \\
\hline
\end{tabular}

\begin{tabular}{|c|c|c|c|c|c|}
\hline Enthesis & Side & Variables & $\begin{array}{l}\text { Measurement } \\
\text { agreement }\end{array}$ & kappa & $\mathbf{p}$ \\
\hline \multirow{8}{*}{$\begin{array}{l}\text { Plantar } \\
\text { fascia }\end{array}$} & \multirow{4}{*}{$\mathrm{R}$} & GS - SP & fair & 0.306 & 0.002 \\
\hline & & $P D-S P$ & moderate & 0.408 & 0.001 \\
\hline & & $G S-E P$ & moderate & 0.481 & 0.001 \\
\hline & & $P D-E P$ & fair & 0.375 & 0.001 \\
\hline & \multirow{4}{*}{ L } & $\mathrm{GS}-\mathrm{SP}$ & moderate & 0.404 & 0.001 \\
\hline & & $P D-S P$ & moderate & 0.586 & 0.001 \\
\hline & & GS - EP & moderate & 0.482 & 0.001 \\
\hline & & $P D-E P$ & moderate & 0.589 & 0.001 \\
\hline \multirow{8}{*}{$\begin{array}{l}\text { Quadri- } \\
\text { ceps } \\
\text { tendon }\end{array}$} & \multirow{4}{*}{$\mathrm{R}$} & $G S-S P$ & moderate & 0.408 & 0.001 \\
\hline & & $P D-S P$ & moderate & 0.452 & 0.001 \\
\hline & & GS - EP & moderate & 0.653 & 0.001 \\
\hline & & $P D-E P$ & fair & 0.381 & 0.001 \\
\hline & \multirow{4}{*}{$\mathrm{L}$} & $G S-S P$ & moderate & 0.443 & 0.001 \\
\hline & & $P D-S P$ & moderate & 0.472 & 0.001 \\
\hline & & GS - EP & moderate & 0.653 & 0.001 \\
\hline & & $P D-E P$ & moderate & 0.415 & 0.001 \\
\hline \multirow{6}{*}{$\begin{array}{l}\text { Proximal } \\
\text { patellar } \\
\text { tendon }\end{array}$} & \multirow{3}{*}{$\mathrm{R}$} & $P D-S P$ & moderate & 0.643 & 0.001 \\
\hline & & $G S-E P$ & moderate & 0.489 & 0.001 \\
\hline & & $P D-E P$ & moderate & 0.500 & 0.001 \\
\hline & \multirow{3}{*}{$\mathrm{L}$} & PD - SP & moderate & 0.556 & 0.001 \\
\hline & & GS - EP & moderate & 0.550 & 0.001 \\
\hline & & $P D-E P$ & moderate & 0.583 & 0.001 \\
\hline
\end{tabular}




\begin{tabular}{|c|c|c|c|c|c|}
\hline Enthesis & Side & Variables & $\begin{array}{l}\text { Measurement } \\
\text { agreement }\end{array}$ & kappa & p \\
\hline \multirow{8}{*}{$\begin{array}{l}\text { Distal } \\
\text { patellar } \\
\text { tendon }\end{array}$} & \multirow{4}{*}{ R } & $\mathrm{GS}-\mathrm{SP}$ & fair & 0.212 & 0.014 \\
\hline & & $P D-S P$ & fair & 0.394 & 0.001 \\
\hline & & $\mathrm{GS}-\mathrm{EP}$ & fair & 0.394 & 0.001 \\
\hline & & $P D-E P$ & fair & 0.306 & 0.003 \\
\hline & \multirow{4}{*}{ L } & $\mathrm{GS}-\mathrm{SP}$ & fair & 0.238 & 0.008 \\
\hline & & $P D-S P$ & moderate & 0.510 & 0.001 \\
\hline & & $\mathrm{GS}-\mathrm{EP}$ & moderate & 0.407 & 0.001 \\
\hline & & $P D-E P$ & moderate & 0.486 & 0.001 \\
\hline \multirow{8}{*}{$\begin{array}{l}\text { Flexor } \\
\text { tendons } \\
\text { of the } \\
\text { hand }\end{array}$} & \multirow{4}{*}{$\mathrm{R}$} & $\mathrm{GS}-\mathrm{SP}$ & poor & 0.162 & 0.024 \\
\hline & & $P D-S P$ & moderate & 0.461 & 0.001 \\
\hline & & $\mathrm{GS}-\mathrm{EP}$ & moderate & 0.508 & 0.001 \\
\hline & & $P D-E P$ & moderate & 0.524 & 0.001 \\
\hline & \multirow{4}{*}{$\mathrm{L}$} & $\mathrm{GS}-\mathrm{SP}$ & poor & 0.197 & 0.003 \\
\hline & & $P D-S P$ & moderate & 0.415 & 0.001 \\
\hline & & $\mathrm{GS}-\mathrm{EP}$ & moderate & 0.619 & 0.001 \\
\hline & & $P D-E P$ & moderate & 0.414 & 0.001 \\
\hline \multirow{6}{*}{$\begin{array}{l}\text { Extensor } \\
\text { tendons } \\
\text { of the } \\
\text { hand }\end{array}$} & \multirow{4}{*}{$\mathrm{R}$} & $\mathrm{GS}-\mathrm{SP}$ & fair & 0.352 & 0.001 \\
\hline & & $P D-S P$ & fair & 0.307 & 0.002 \\
\hline & & $\mathrm{GS}-\mathrm{EP}$ & moderate & 0.533 & 0.001 \\
\hline & & $P D-E P$ & moderate & 0.654 & 0.001 \\
\hline & \multirow{2}{*}{$\mathrm{L}$} & $P D-S P$ & moderate & 0.450 & 0.001 \\
\hline & & $P D-E P$ & moderate & 0.600 & 0.001 \\
\hline
\end{tabular}

$E P$ - elicited pain; GS - grey scale; $L$ - left; $P D$ - power Doppler; $R$-right; $S P$ - spontaneous pain

TABLE 8. Prevalence of ultrasound detected enthesitis in asymptomatic entheses per entheseal site

\begin{tabular}{|c|c|c|}
\hline $\begin{array}{c}\text { Asymptomatic } \\
\text { entheses (SP = 0 + EP } \\
\text { = 0) }\end{array}$ & $\begin{array}{c}\text { GS } \\
\text { abnormalities }\end{array}$ & $\begin{array}{c}\text { PD } \\
\text { abnormalities }\end{array}$ \\
\hline Achilles tendon (64) & $35(54.68 \%)$ & $4(6.25 \%)$ \\
\hline Plantar fascia (133) & $19(14.28 \%)$ & $3(2.25 \%)$ \\
\hline $\begin{array}{c}\text { Quadriceps tendon } \\
\text { (86) }\end{array}$ & $22(25.58 \%)$ & $2(2.32 \%)$ \\
\hline $\begin{array}{c}\text { Proximal patellar } \\
\text { tendon (142) }\end{array}$ & $18(12.67 \%)$ & $4(2.81 \%)$ \\
\hline $\begin{array}{c}\text { Distal patellar tendon } \\
\text { (111) }\end{array}$ & $37(33.33 \%)$ & $7(6.30 \%)$ \\
\hline Triceps tendon (132) & $23(17.42 \%)$ & $2(1.51 \%)$ \\
\hline $\begin{array}{c}\text { Flexor tendons of the } \\
\text { hand (143) }\end{array}$ & $19(13.28 \%)$ & $2(1.39 \%)$ \\
\hline $\begin{array}{c}\text { Extensor tendons of } \\
\text { the hand (135) }\end{array}$ & $27(20 \%)$ & $11(8.14 \%)$ \\
\hline
\end{tabular}

EP - elicited pain, GS - gray scale, PD - power Doppler,

$S P$ - spontaneous pain

\section{DISCUSSIONS}

Enthesitis was detected in higher percentages in patients by ultrasound rather than asking the patient questions about the entheseal pain and by clinical examination, a result that was predictable to some extent, considering the scientific literature [24,25,27].

Ultrasound GS signs of enthesitis were detected in up to $54.68 \%$ of the asymptomatic (after both medical history and physical examination) Achilles ten- don insertions, while PD signal was detected in lower rates, up to $8.14 \%$ in the extensor tendons of the hand insertions.

In all evaluated tendon insertions, ultrasound had a better rate of enthesitis detection than the clinical evaluation alone. The clinical and ultrasound results were better correlated in the flexor tendons of the hands, in regard to the detection of $\mathrm{PD}$, proportional to pain, both spontaneous and elicited. Lower rates of asymptomatic signs of enthesitis were found in points of high mechanical stress and pressure, like the triceps tendon insertion; the olecranon process being prone to mechanical stress and repeated trauma, the Achilles tendon, where it is more likely for an entheseal calcification do cause pain, but interestingly, not the plantar fascia as well [28].

Considering that a highly active disease was one of the inclusion criteria, a comparison with the ultrasound findings in a low disease activity patient is an obvious checkpoint further along this study, but at this point, unavailable.

Also, taking into account the 6 year period measurement and the delay in diagnosing $\mathrm{SpA}$, leading to physical deficits, worse quality of life, with phychological consequences and increased economic burden, a tool for early diagnosis should be considered in every day practice, ultrasonography and MRI being the go-to methods. The choice remains to be wheightened according to the advantages and limitations of each method, while consideringthat an early diagnosis per-se offers better prognosis, adding up to better treatment responses [29,30].

The limitations of the study are mostly with reference to the small number of patients at this point in the study. For better correlations regarding more tendons and the entire lot, more patients are being enrolled in the study. Also, the patients were evaluated dynamically, after undergoing therapy and obtaining variable rates of lower disease activity than at baseline.

Degenerative entheseal abnormalities, mostly GS, could generate false-positive US results, while the clinical examination could also be influenced by the possible overlap with fibromyalgia. According to recent studies, the prevalence of fibromyalgia has been reported in $4-25 \%$ of SpA patients, mostly females, compared to $2-8 \%$ in the general population. This leads to significantly higher patient-reported outcome rates and ASDAS score in patients with concomitant fibromyalgia [31]. A further research direction on this subject is examining enthesitis patterns in healthy subjects. 
This is an ongoing study (prospective research), which will focus on the evaluation the clinical and ultrasound changes after 6 months and 12 months of bDMARD versus non-bDMARD therapy, compared to baseline.

\section{CONCLUSIONS}

Enthesitis is a hallmark feature of SpA, preceeding or leading to structural damage in the pathogenetic course of events. The clinical examination itself offers limited information regarding structural abnormalities, local inflammatory status and a weak differentiation between entheseal or referred pain, imaging techniques, such as ultrasound and MRI, having the capacity of completing a well conducted medical history and clinical examination. Between the two, ultrasound is more compatible with the clinical, everyday practice, while MRI could give more accurate information, but limited to a certain segment.

There is a low correlation between entheseal pain and ultrasound GS and/or PD enthesitis, especially in the Achilles and distal patellar tendons.

The significant difference between the clinical findings, either spontaneous or elicited pain, and the ultrasound-detected entheseal involvement, both GS and $\mathrm{PD}$, proves the existence of a gap in diagnosing enthesitis and, therefore, establishing the full extent

\section{REFERENCES}

1. Barnett $R$, Ingram $T$, Sengupta R. Axial spondyloarthritis 10 years on: still looking for the lost tribe. Rheumatology (Oxford). 2020;59(4):iv25-iv37.

2. Ionescu R. Spondilartropatii seronegative. In: Esentialul in reumatologie. Ed Amaltea, 2006;281-293.

3. López-Medina C, Molto A, Claudepierre P, Dougados M. Clinical manifestations, disease activity and disease burden of radiographic versus non-radiographic axial spondyloarthritis over 5 years of followup in the DESIR cohort. Ann Rheum Dis. 2020;79(2):209-216.

4. Opris-Belinski D. Conceptul de spondiloartrite in Reumatologie Curs Universitar. Ed Universitara "Carol Davila" 2017;179-181.

5. Wendling D, Prati C. Flare in axial spondyloarthritis. The dark side of the outcome. Ann Rheum Dis. 2016;75:950-951.

6. Gossec L, Portier A, Landewé R, et al. Preliminary definitions of 'flare' in axial spondyloarthritis, based on pain, BASDAI and ASDASCRP: an ASAS initiative. Ann Rheum Dis. 2016;75:991-996.

7. Garrett S, Jenkinson T, Kennedy LG, et al. A new approach to defining disease status in ankylosing spondylitis: the Bath Ankylosing Spondylitis Disease Activity Index. J Rheumatol. 1994;21(12):2286-2291.

8. Machado P, Landewé R, Lie E, et al. Assessment of SpondyloArthritis international Society. Ankylosing Spondylitis Disease Activity Score (ASDAS): defining cut-off values for disease activity states and improvement scores. Ann Rheum Dis. 2011;70(1):47-53.

9. Wang R, Ward MM. Epidemiology of axial spondyloarthritis: an update. Curr Opin Rheumatol. 2018;30(2):137-143.

10. de Winter JJ, van Mens LJ, van der Heijde D, et al. Prevalence of peripheral and extra-articular disease in ankylosing spondylitis of SpA in each patient. These findings come in support to the already declared need for an imagistic enthesitis score in the diagnostic process of SpA.

Entheseal ultrasonography could be useful for the screening of patients with chronic low back pain or unclassified arthritis, in order to diagnose SpA early, before the structural damage formation occurs and it may help in establishing the full extent of the disease, but also to design a more personalized therapeutic plan, even more so, considering the need to distinguish between the disease-related patient-reported outcomes and the possible intricate degenerative of psychosomatic pathology.

\section{Acknowledgements}

This paper and the research behind it would not have been possible without the exceptional support of the Rheumatology physicians and trainees from the Internal Medicine and Rheumatology Departments of the "Sfânta Maria" Clinical Hospital and "Dr. I. Cantacuzino" Clinical Hospital, in Bucharest, who referred patients that matched the study inclusion criteria.

The authors would also like to show our deepest gratitude to Claudiu C. Popescu, who previously offered a pattern that helped the elaboration of this paper.

Conflict of interest: none declared Financial support: none declared

versus non-radiographic axial spondyloarthritis: a meta-analysis. Arthritis Res Ther. 2016;18(1):196.

11. Poddubnyy D, Sieper J. Radiographic progression in ankylosing spondylitis/axial spondyloarthritis: how fast and how clinically meaningful? Curr Opin Rheumatol. 2012;24:363-369.

12. Sari I, Lee S, Tomlinson G, et al. Factors Predictive of Radiographic Progression in Ankylosing Spondylitis. Arthritis Care Res (Hoboken). 2019 Nov. Online ahead of print.

13. Benjamin M, McGonagle D. The enthesis organ concept and its relevance to the spondyloarthropathies. Adv Exp Med Biol. 2009;649:57-70.

14. McGonagle D, Stockwin L, Isaacs J, Emery P. An enthesitis based model for the pathogenesis of spondyloarthropathy: additive effects of microbial adjuvant and biomechanical factors at disease sites. $J$ Rheumatol. 2001;28:2155-2159.

15. Watad A, Cuthbert RJ, Amital H, McGonagle D. Enthesitis: Much More Than Focal Insertion Point Inflammation. Curr Rheumatol Rep. 2018;20(7):41.

16. McGonagle D, Marzo-Ortega H, O'Connor P, et al. Histological assessment of the early enthesitis lesion in spondyloarthropathy. Ann Rheum Dis. 2002;61:534-537.

17. Kehl AS, Corr M, Weisman MH, et al. Enthesitis - New Insights Into Pathogenesis, Diagnostic Modalities, and Treatment. Arthritis Rheumatol. 2016;68(2):312-322.

18. Weiss PF. Evaluation and treatment of enthesitis-related arthritis. Curr Med Lit Rheumatol. 2013;32:33-41.

19. Feldtkeller E, Khan MA, van der Heijde D, et al. Age at disease onset and diagnosis delay in HLA-B27 negative vs. positive patients with ankylosing spondylitis. Rheumatol Int. 2003;23:61-66. 
20. Rosenbaum JT, Pisenti L, Park Y, Howard RA. Insight into the quality of life of patients with ankylosing spondylitis: real-world data from a US-based life impact survey. Rheumatol Ther. 2019;6:353-367.

21. Rudwaleit M, van der Heijde D, Landewé R, et al. The development of Assessment of SpondyloArthritis international Society classification criteria for axial spondyloarthritis (part II): validation and final selection. Ann Rheum Dis. 2009;68(6):777-783.

22. Rudwaleit $M$, van der Heijde $D$, Landewé $R$, et al. The Assessment of SpondyloArthritis International Society classification criteria for peripheral spondyloarthritis and for spondyloarthritis in general. Ann Rheum Dis. 2011;70(1):25-31.

23. D'Agostino MA, Olivieri I. Enthesitis. Best Pract Res Clin Rheumatol. 2006:20:473-486

24. Ruta S, Gutierrez M, Pena C, et al. Prevalence of subclinical enthesopathy in patients with spondyloarthropathy: an ultrasound study. J Clin Rheumatol. 2011;17:18-22.

25. D'Agostino MA, Said-Nahal R, Hacquard-Bouder C, et al. Assessment of peripheral enthesitis in the spondylarthropathies by ultrasonography combined with power Doppler: a cross-sectional study. Arthritis Rheum. 2003;48:523-533.
26. Gisev, T.F., Bell, S.J., Chen, T.F. Interrater agreement and interrater reliability: Key concepts, approaches, and applications. RSAP. 2013;9(3):330-338

27. Mlauser AS, Wipfler E, Dejaco C, et al. Diagnostic values of history and clinical examination to predict ultrasound signs of chronic and acute enthesitis. Clin Exp Rheumatol.2008;26:548-553.

28. Di Matteo A, Filippucci E, Cipolletta E, et al. Entheseal Involvement in Asymptomatic Healthy Subjects: Prevalence and Distribution of the Ultrasound Elementary Lesions of Enthesitis, with a Particular Focus on Those Indicating "Active" Inflammation [abstract]. Arthritis Rheumatol 2018;70(10).

29. Seo MR, Baek HL, Yoon HH, et al. Delayed diagnosis is linked to worse outcomes and unfavourable treatment responses in patients with axial spondyloarthritis. Clin Rheumatol. 2015;34:1397-1405.

30. Yi E, Ahuja A, Rajput T, et al. Clinical, economic, and humanistic burden associated with delayed diagnosis of axial spondyloarthritis: a systematic review. Rheumatol Ther. 2020;7:65-87.

31. Mease PJ. Fibromyalgia, a missed comorbidity in spondyloarthritis: prevalence and impact on assessment and treatment. Curr Opin Rheumatol. 2017;29(4):304-310 